Hautarzt 2018 $69: 631-640$

https://doi.org/10.1007/s00105-018-4229-z

Online publiziert: 13. Juli 2018

(c) Der/die Autor(en) 2018

CrossMark

Franz J. Legat

Universitätsklinik für Dermatologie und Venerologie, Medizinische Universität Graz, Graz, Österreich

\title{
Stellenwert der Phototherapie in der Behandlung des chronischen Pruritus
}

ligem UVA-1 sowie mit Excimer-Laser $(308 \mathrm{~nm})$ haben in die Phototherapie Einzug gehalten und ein breites Einsatzfeld gefunden $[3,4]$.

Viele der Hauterkrankungen, die sich durch UV-Strahlung bessern lassen, gehen mit chronischem Pruritus, also Pruritus für 6 Wochen oder länger, einher. Bei Psoriasis, atopischer Dermatitis oder Mycosis fungoides, die mit Photo(chemo)therapie sehr erfolgreich behandelt werden, bessern sich durch die UV-Bestrahlung aber nicht nur die Hautveränderungen, sondern auch der quälende Pruritus. Es konnte aber auch gezeigt werden, dass nicht nur chronischer Pruritus bei primären Hauterkrankungen (Gruppe 1 der IFSI[International Forum for the Study of Itch]-Klassifikation; [5]), sondern auch chronischer Pruritus bei primär unveränderter Haut (Gruppe 2 der IFSI-Klassifikation) wie z. B. bei chronischer Niereninsuffizienz, (cholestatischen) Lebererkrankungen sowie hämatologischen Erkrankungen durch die Phototherapie reduziert werden kann. Nicht zuletzt sprechen auch Krankheitszustände, bei denen chronischer Pruritus und massives Kratzen zu sekundären Kratzeffloreszenzen geführt haben (Gruppe 3 der IFSI-Klassifikation), positiv auf eine UV-Therapie an.

$\mathrm{Zu}$ letzterer Gruppe gehört auch die chronische Prurigo mit ihren klinischen Formen (papulöser, nodulärer, plaqueförmiger oder umbilikal-ulzeröser-Typ; [6]). Diese hochchronische Erkrankung, die sich nach heutiger Expertenmeinung durch eine neuronale Sensibilisierung und die Entstehung eines Juck-Kratz-Zyklus entwickelt, zeigt sich meist äußerst

hartnäckig gegenüber den verschiedenen heute verfügbaren Therapien. Auch bei der Erkrankung „chronische Prurigo“ haben sich die verschiedenen Varianten der Photo(chemo)therapie als sehr erfolgreich gezeigt.

\section{1) Bei chronischer Prurigo sind die verschiedenen Varianten der Photo(chemo)therapie sehr erfolgreich}

Wenn sich bei chronischem Pruritus keine zugrunde liegende Ursache findet, die entweder vollständig beseitigt oder zumindest signifikant verbessert werden kann, ist mit einem Verschwinden des Pruritus nicht $\mathrm{zu}$ rechnen. Aber auch nach Behandlung der Ursache bleibt oft chronischer Pruritus bestehen und stellt für die Betroffenen eine signifikante Einschränkung der Lebensqualität dar. In einer epidemiologischen Studie in Deutschland konnte gezeigt werden, dass bis zu 13,5\% der Befragten zum Zeitpunkt der Befragung an chronischem Pruritus leiden und die Lebenszeitprävalenz sogar $22 \%$ betrug [7]. Dies zeigt den großen Bedarf an wirksamen Behandlungen des chronischen Pruritus in der Bevölkerung.

Zurzeit gibt es noch keine zugelassenen Medikamente gegen chronischen Pruritus. Neben Antihistaminika werden in ausgeprägten Fällen von chronischem Pruritus daher oft Medikamente aus anderen Bereichen der Medizin wie Neurologie und Psychiatrie oder innere Medizin eingesetzt, bei denen beobachtet wurde, dass sie als „Nebenwirkung“ beglei- re Entwicklungen wie die UV-Therapien mit Schmalband-UVB oder langwel- 
tenden Pruritus hemmen konnten ([8]; Zeidler et al. 2018, in diesem Heft).

In den letzten Jahren haben intensive Forschungsanstrengungen auf dem Gebiet des Pruritus signifikante Fortschritte gebracht und mehrere Mediatoren und Angriffspunkte als Ansatzpunkte für die Behandlung des Pruritus identifiziert ([9]; Pereira et al. 2018, in diesem Heft). Bereits jetzt und in den kommenden Jahren werden wir daher die Ergebnisse einer Reihe von klinischen Prüfungen von „neuen Medikamenten“ gegen (chronischen) Pruritus sehen (Zeidler et al. 2018, in diesem Heft). Bis zur Zulassung entsprechend wirksamer Medikamente gegen chronischen Pruritus und deren Einsatz in der täglichen Praxis werden aber noch einige Jahre vergehen.

\section{Phototherapie in der Behandlung von chronischem Pruritus}

Die Photo(chemo)therapie, in weiterer Folge kurz als „Phototherapie“ zusammengefasst, ist bereits seit Langem verfügbar und immer noch eine wichtige Säule in der Behandlung von entzündlichen Hauterkrankungen wie Psoriasis und atopischer Dermatitis - trotz der Einführung der Biologika zur Behandlung dieser Erkrankungen. Die Phototherapie führt nicht nur zu einer Besserung der Hautveränderungen, sondern zeigt auch einen sehr gut hemmenden Effekt auf den oft quälenden Pruritus bei diesen Erkrankungen [10, 11]. Viele andere Formen des chronischen Pruritus mit und ohne primäre oder sekundäre Hautveränderungen sprechen ebenfalls sehr gut auf Phototherapie an [12]. Neben der Reduktion des Pruritus werden durch die Phototherapie auch pruriginöse Hautveränderungen oder sekundäre Kratzeffloreszenzen gebessert oder zur Abheilung gebracht.

In der AWMF(Arbeitsgemeinschaft der Wissenschaftlichen Medizinischen Fachgesellschaften)-Leitlinie „Diagnostik und Therapie des chronischen Pruritus" [10] konnte unter den teilnehmenden Experten ein Konsens zur UVPhototherapie bei chronischem Pruritus erzielt werden, der wie folgt lautet: „Eine UV-Phototherapie kann bei chronischem
Pruritus (CP), bei entzündlichen Dermatosen und chronischen Kratzläsionen empfohlen und bei CP bei ausgewählten inneren Erkrankungen erwogen werden."

Auch wenn der genaue Wirkmechanismus, wie die UV-Strahlung der Sonne oder einer Phototherapie zur Hemmung des Pruritus führt, derzeit noch nicht genau bekannt ist, wird die Phototherapie seit Jahrzehnten gegen juckende Hauterkrankungen und chronischen Pruritus generell eingesetzt, und die klinische Erfahrung belegt ihre Wirkung. Von entscheidender Bedeutung für die Wirkung der UV-Strahlung ist neben der Wellenlänge v. a. die applizierte UVDosis. So führen hohe UV-Dosen, die eine Hautrötung und Entzündung verursachen, sogar zur Auslösung oder signifikanten Zunahme des bestehenden Pruritus, während wiederholte UV-Dosen unter der Rötungsschwelle Pruritushemmend wirken.

\section{》) Wichtig für die Wirkung} der UV-Strahlung ist neben der Wellenlänge die applizierte UVDosis

Entscheidend ist auch die zugrunde liegende Erkrankung des Patienten. Während bei entzündlichen Hauterkrankungen wie Psoriasis, atopischer Dermatitis oder exanthematischem Lichen planus die antiinflammatorische Wirkung der Phototherapie möglicherweise einen wichtigen Faktor für die „begleitende" Reduktion des Pruritus darstellt, scheinen bei chronischem Pruritus im Rahmen von systemischen Erkrankungen wie chronischer Niereninsuffizienz, (cholestatischen) Lebererkrankungen oder hämatologischen Erkrankungen andere bisher nicht aufgeklärte Mechanismen für die antipruritische Wirkung der Phototherapie verantwortlich $\mathrm{zu}$ sein.

Wenn UV-Strahlung auf die Haut trifft, so erreicht sie v. a. die oberen Hautschichten. Dabei wirkt das kurzwellige UVB (280-320 nm) v.a. auf die Epidermis und die obere papilläre Dermis, während das längerwellige UVA (320-400 nm) bis in die retikulä- re Dermis vordringt. In diesen oberen Hautschichten finden sich die residenten Zellen der Epidermis und Dermis wie Keratinozyten, Langerhans-Zellen, Melanozyten, Fibroblasten, Endothelzellen der Gefäße, Schweißdrüsenzellen und Haarfollikelzellen. Darüber hinaus werden auch Zellen, die in die Haut einwandern, wie Mastzellen, eosinophile Granulozyten, Leukozyten, dendritische Zellen und Lymphozyten von einstrahlendem UV-Licht getroffen. Aber nicht nur Zellen sind Zielstrukturen der UVStrahlung, sondern auch Bestandteile des Stratum corneum wie die trans-Urocaninsäure, die durch UVB-Strahlung in ihre cis-Form überführt wird [13].

Oft vergessen, aber für das Symptom "Pruritus" von entscheidender Bedeutung, sind die Nerven der Haut. Sensorische Nervenfasern durchziehen mit einem dichten dreidimensionalen Netzwerk die gesamte Haut und bilden unter der dermoepidermalen Junktionszone ein subepidermales Nervengeflecht, von dem aus feine sensorische Nervenfasern durch die Basalmembran in die Epidermis einwachsen und zwischen den Keratinozyten bis zum Stratum corneum vordringen und sich auf ihrem Weg vielfältigverzweigen. Diese sensorischen, feinen, myelinfreien C-Fasern in der Epidermis und gering myelinisierten A-delta-Fasern in der oberen Dermis sind somit ebenfalls im Zielbereich der auf die Haut eintreffenden UV-Strahlung. In dieser Gruppe von C- und A-delta-Fasern finden sich jene sensorische Nervenfasern, die Pruritussignale aufnehmen und nach zentral weiterleiten [14].

UV-Strahlung kann also auf zelluläre, nichtzelluläre und neuronale Strukturen treffen und dort eine Vielzahl von Reaktionen auslösen. Ein wesentlicher Effekt der UV-Strahlung ist die Wirkung auf die DNA der Zellen in der Haut und die daraus resultierende lokale und systemische Immunsuppression, bei der dendritische Zellen, zahlreiche lösliche Mediatoren und die Ausbildung von regulatorischen T-Zellen eine wichtige Rolle spielen [15]. Bei der Vermittlung der lokalen und systemischen Immunsuppression spielen aber auch die UV-Effekte auf das sensorische Nervensystem der Haut und die Freisetzung verschie- 
dener Mediatoren wie die Neuropeptide „substance P“ (SP) und „calcitonin gene-related peptide“ (CGRP) aus sensorischen Nerven sowie der Einfluss anderer UV-induzierter Mediatoren aus residenten und eingewanderten Hautzellen und Bestandteilen des Stratum corneum (z.B. cis-Urocaninsäure) eine nicht minder wichtige Rolle $[16,17]$. Welchen Beitrag die UV-induzierte Immunsuppression bei der Vermittlung der antipruritischen Wirkung der Phototherapie leistet, bleibt vorerst unbeantwortet. Jedenfalls ist eine systemische Unterdrückung des Immunsystems wie z. B. durch Glukokortikoide, Cyclosporin oder Methotrexat bei generalisiertem Pruritus unterschiedlicher Genese mitunter sehr gut wirksam [18].

\section{》) Eine systemische Unter- drückung des Immunsystems ist bei generalisiertem Pruritus mitunter sehr wirksam}

Wichtig in der Vermittlung der antipruritischen Wirkung der Phototherapie könnten aber auch direkte UV-Einflüsse auf die sensorischen Nerven, wie z.B. eine „Desensibilisierung" gegenüber bestimmten pruritogenen Reizen, sein. Andererseits könnte auch die Beeinflussung von Strukturen und $\mathrm{Me}$ diatoren, die bei der Auslösung und Weiterleitung sowie bei der Unterhaltung des chronischen "Juckreizes“ eine Rolle spielen, einen wesentlichen Beitrag liefern. Solche Mediatoren sind z. B. Interleukin(IL)-4, IL-13 und IL-31 aus Lymphozyten, Mastzellen oder Eosinophilen, die beim chronischen Pruritus der atopischen Dermatitis eine wichtige Rolle spielen. Auch der Einfluss der UVStrahlung auf andere Mediatoren wie „nerve growth factor“ (NGF), Tryptase, „substance $\mathrm{P}^{\text {“, „calcitonin gene-related }}$ peptide“ (CGRP) und „thymic stromal lymphopoetin“ (TSLP), die aus infiltrierenden und residenten Zellen der Haut und Nerven freigesetzt werden, könnten einen Betrag zur antipruritischen Wirkung liefern.

Wichtige Beobachtungen zur antipruritischen Wirkung der Phototherapie

Hautarzt 2018 -69:631-640 https://doi.org/10.1007/s00105-018-4229-z

(c) Der/die Autor(en) 2018

\section{F. J. Legat}

\section{Stellenwert der Phototherapie in der Behandlung des chronischen Pruritus}

\section{Zusammenfassung}

Die Phototherapie und Photochemotherapie (PUVA) sind wichtige Säulen in der Behandlung von entzündlichen Hauterkrankungen wie Psoriasis und atopischer Dermatitis sowie des kutanen T-Zell-Lymphoms (z. B Mycosis fungoides/Sezary-Syndrom). Viele dieser Hauterkrankungen gehen mit quälendem Pruritus einher. Auch bei systemischen Erkrankungen der Niere und Leber sowie des endokrinen und blutbildenden Systems kann Pruritus ein belastendes Symptom der betroffenen Patienten sein. Die Behandlung mit ultraviolettem Licht kann nicht nur spezifische Hautveränderungen bessern, sondern auch den Pruritus vieler Hautkrankheiten sowie vieler systemischer Erkrankungen reduzieren. Die meist sehr gute antipruritische Wirkung, die bekannten und bei richtiger Durchführung geringen Nebenwirkungen sowie die Möglichkeit des Einsatzes bei Erwachsenen jeden Alters, Schwangeren/Stillenden und unter bestimmten Bedingungen auch bei Kindern machen die Phototherapie zu einer wertvollen Behandlungsmöglichkeit bei chronischem Pruritus unterschiedlicher Ursache. Der Einsatz der Phototherapie sollte daher früh erwogen werden, wenn lokaltherapeutische Maßnahmen nicht ausreichen, um den Pruritus zu lindern.

Schlüsselwörter

Chronische Prurigo · UVB · PUVA · Entzündliche Hautkrankheiten · Systemische Erkrankungen

\section{Importance of phototherapy in the treatment of chronic pruritus}

\begin{abstract}
Phototherapy and photochemotherapy (PUVA) are important treatment modalities in inflammatory skin diseases such as psoriasis and atopic dermatitis as well as in cutaneous T-cell lymphoma (e.g., mycosis fungoides/Sezary syndrome). Many of these skin diseases are accompanied by distracting pruritus. In addition, patients may suffer from intense pruritus in systemic diseases of the kidney and liver as well as of the endocrine and hematopoietic system. UVlight during phototherapy is capable of not only improving the inflammatory skin lesions but also of reducing the pruritus in skin and systemic diseases. The significant antipruritic
\end{abstract}

effect, the usually low rate of well-known side effects, as well as the possibility to treat adults of any age, pregnant and lactating women, and under certain circumstances also children, make phototherapy a valuable treatment option for pruritus of various origin. Thus, the use of phototherapy should be considered early in the course of antipruritic therapy, when topical treatment modalities are insufficient to significantly improve pruritus.

\section{Keywords}

Chronic prurigo $\cdot$ UVB $\cdot$ PUVA · Inflammatory skin diseases $\cdot$ Systemic diseases machten Gilchrest et al. bei der UVBTherapie von hämodialysepflichtigen, niereninsuffizienten Patienten mit chronischem Pruritus. Bei 9 von 10 Patienten verschwand der chronische Pruritus bei 2-mal wöchentlicher Ganzkörper-UVBTherapie. Eine wiederholte Bestrahlung nur des halben Körpers führte zum Verschwinden des Pruritus auch auf der unbestrahlten Körperhälfte [19, 20]. Der antipruritische Effekt setzte aber nicht unmittelbar, sondern zeitverzögert nach erst etwa 2 Wochen ein, wobei bei einer UV-Therapie 3-mal pro Woche die an- tipruritische Wirkung früher eintrat als bei UV-Therapie nur 1-mal pro Woche. Aus diesen Untersuchungen folgerten die Autoren, dass durch wiederholte UVBBestrahlung der Haut ein bis heute unbekannter Faktor entsteht, der eine systemische antipruritische Wirkung bei den Hämodialysepatienten mit chronischem Pruritus bewirkt. Sie verwiesen aber auch auf andere Beispiele von juckenden inflammatorischen Hauterkrankungen wie atopische Dermatitis, Psoriasis, Mycosis fungoides und Pityriasis rosea, bei denen UVB oder PUVA zu einer loka- 
Hier steht eine Anzeige.

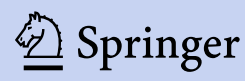


Hier steht eine Anzeige.

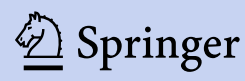


Tab. 1 Phototherapeutische Optionen bei ausgewählten Krankheitszuständen mit chronischem Pruritus

\begin{tabular}{|c|c|c|c|}
\hline Photo(chemo)therapien & UVB-NB/UVB-BB & UVA-1 & PUVA/Bade-PUVA \\
\hline \multicolumn{4}{|c|}{ CP bei primären Hauteffloreszenzen (IFSI-Gruppe 1) } \\
\hline Atopische Dermatitis & $++/+$ & ++ & $++/++$ \\
\hline Psoriasis vulgaris & $++/+$ & - & $++/++$ \\
\hline $\begin{array}{l}\text { Mycosis fungoides } \\
\text { Sezary-Syndrom }\end{array}$ & $\begin{array}{l}+/+ \\
-/-\end{array}$ & $\begin{array}{l}- \\
-\end{array}$ & $\begin{array}{l}++/+ \\
++/+\end{array}$ \\
\hline Urticaria pigmentosa & $-/-$ & ++ & $++/++$ \\
\hline \multicolumn{4}{|c|}{ CP ohne primäre Hauteffloreszenzen (IFSI-Gruppe 2) } \\
\hline Chronische Niereninsuffizienz & $++/++$ & - & - \\
\hline $\begin{array}{l}\text { Hepatischer Pruritus (Cholestase } \\
\text { inklusive Schwangerschaftscho- } \\
\text { lestase) }\end{array}$ & $+/+$ & - & - \\
\hline Polycythaemia vera & $++/++$ & - & $+/+$ \\
\hline Hodgkin Lymphom & $+/+$ & - & $+/+$ \\
\hline Chronisch idiopathischer Pruritus & $+/+$ & + & $-/+$ \\
\hline \multicolumn{4}{|c|}{ Chronische Prurigo und CP mit sekundären Kratzeffloreszenzen (IFSI-Gruppe 3) } \\
\hline Chronische Prurigo & $++/++$ & + & $++/++$ \\
\hline $\begin{array}{l}\text { CP mit sekundären Kratzefflores- } \\
\text { zenzen }\end{array}$ & $++/++$ & + & $+/+$ \\
\hline \multicolumn{4}{|c|}{ 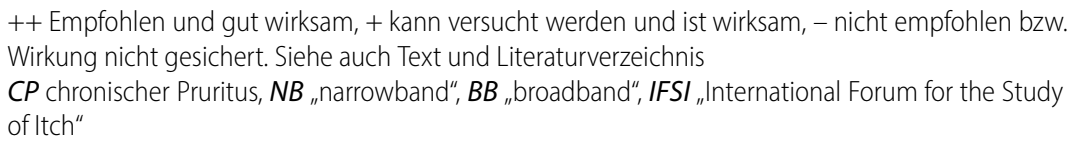 } \\
\hline
\end{tabular}

len, nichtsystemischen Unterdrückung des Pruritus geführt hat [23]. In einer Studie bei atopischer Dermatitis konnten die Patienten bei einer Therapie mit $\mathrm{NB}($,narrowband“)-UVB auf der einen Körperhälfte und UVA-1 auf der anderen im Verlauf einen subjektiven Unterschied in der Pruritusintensität zwischen den beiden Seiten bemerken [21], was ebenfalls für eine lokale Unterdrückung des Pruritus spricht. Allerdings kann bei einer Halbseiten-UV-Therapie auch nie ausgeschlossen werden, dass auch systemische Pruritus-unterdrückende Mechanismen mit am Werke sind. Insgesamt sind also wahrscheinlich die verwendete UV-Strahlung, die bestrahlte Fläche sowie die zugrunde liegende Erkrankung ausschlaggebend, ob lokale und/oder systemische Mechanismen für die Unterdrückung des Pruritus durch UV-Strahlung zum Tragen kommen.

\section{Phototherapie von chronisch juckenden Haut- und Systemerkrankungen in der täglichen Praxis}

Der Einsatz der Phototherapie und besonders der Photochemotherapie (PUVA) hängt sehr stark von der Ausstattung der jeweiligen Organisationseinheit (Hautklinik, Hautambulatorium oder Hautarztpraxis) und der „Ausbildung in Photodermatologie“ der Ärztinnen und Ärzte ab. Befindet sich eine Phototherapieeinheit in einer Hautklinik oder einem Hautambulatorium und sind die Ärzte in diesen Einrichtungen ausreichend phototherapeutisch geschult worden, so werden diese Ärzte die Phototherapie auch vermehrt für juckende Dermatosen oder Systemerkrankungen in ihrem jeweiligen Arbeitsumfeld der Klinik oder in ihren zukünftigen Praxen einsetzen. Die „Leitlinie für Phototherapie der Deutschen dermatologischen Gesellschaft" (2016) beschreibt sehr klar, welche Voraussetzungen für eine erfolgreiche Phototherapie in Klinik und Praxis gegeben sein müssen, und gibt Hilfestellungen bei der Indikationsstel- lung, Vorbereitung und Durchführung einer Phototherapie [4].

\section{Welche Erkrankungen mit chronischem Pruritus kann man mit Phototherapie behandeln?}

\section{Chronischer Pruritus bei primären Hautveränderungen (Gruppe I der IFSI-Klassifikation)}

Entzündliche Hauterkrankungen wie Psoriasis und atopische Dermatitis, die mit chronischem Pruritus einhergehen, sprechen üblicherweise sehr gut auf die Phototherapie an (-Tab. 1). Bei der Phototherapie der Psoriasis mit Schmalband(NB)-UVB oder PUVA setzt die antipruritische Wirkung meist rascher ein als die sichtbare Abheilung der psoriatischen Hautveränderungen, sofern, wie bereits oben erwähnt, keine zu hohe UV-Dosierung gewählt wird, die den Pruritus sogar verstärken kann [10]. Der Pruritus, wenn vorhanden, scheint eng mit dem Entzündungsgeschehen der Psoriasis verbunden $\mathrm{zu}$ sein. In einer Studie zeigte sich der Pruritus vor Beginn der NB-UVB-Therapie sogar als Indikator für die Zahl der in weiterer Folge notwendigen Bestrahlungen bis zur Abheilung [22]. In dieser Studie bestätigte sich aber auch ein aus der Praxis bekannter Umstand, dass bei der Psoriasis ein erythematogenes UVDosis-Schema rascher zur Abheilung der psoriatischen Effloreszenzen führte als ein suberythematogenes, auch wenn anfänglich der Pruritus dadurch sogar noch verstärkt wurde.

\section{》) Der Pruritus, wenn vorhanden, scheint eng mit dem Entzündungsgeschehen der Psoriasis verbunden zu sein}

Für die atopische Dermatitis ist aber ein suberythematogenes Therapieschema anzuwenden, da es sonst nicht nur zu einer Zunahme des Pruritus, sondern auch der Hautentzündungen kommt. In mehreren Studien ist klar belegt, dass die atopischen Ekzeme und der chronische Pruritus durch alle Formen 


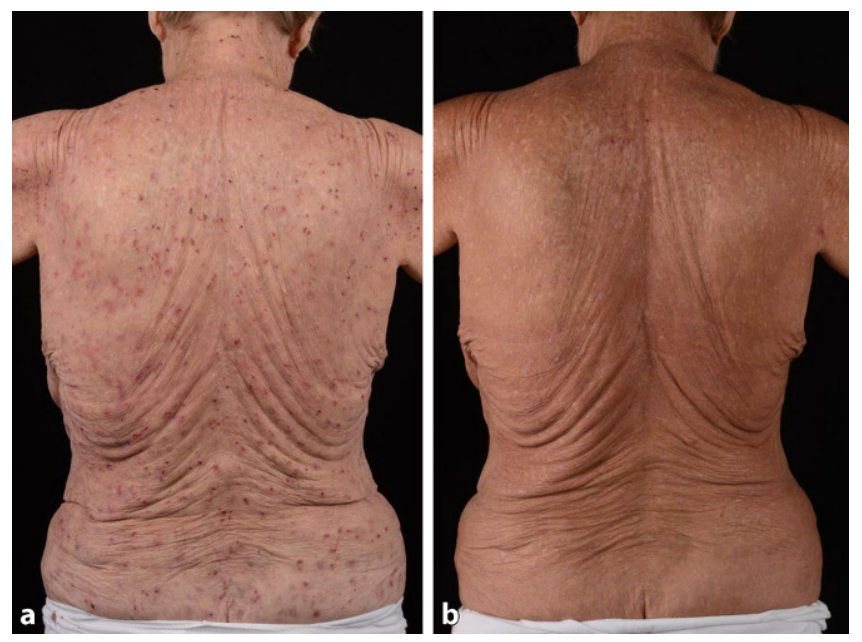

Abb. 1 ॥ 78-jährige Patientin mit chronischer Niereninsuffizienz, monoklonalerGammopathie unbestimmter Signifikanz (MGUS), Diabetes mellitus Typ 2 und Depression a vor und $\mathbf{b}$ nach einer Schmalband(NB)-UVB-Therapie (3-mal/Woche für 12 Wochen, 34 Therapien, kumulative UV-Dosis 47,4 J/cm²). Pruritus (Numeric Rating Scale [NRS] von $0=$ kein Pruritus bis $10=$ stärkster vorstellbarer Pruritus) a vor NB-UVB (10/10), b nach NB-UVB (0/10)

der Phototherapie bei suberythematogener Dosierung gebessert werden [11]. NB-UVB und, wenn verfügbar, UVA-1 („medium dose“), sind derzeit die bevorzugten Phototherapien bei atopischer Dermatitis. PUVA (orale oder Bade-PUVA), kann bei therapieresistenten Fällen oft mit Erfolg eingesetzt werden [4].

Für andere Hauterkrankungen, die mit chronischem Pruritus einhergehen, sind primär auch die empfohlenen UVTherapien zu bevorzugen, da mit der Abheilung der Hautveränderungen meist der chronische Pruritus verschwindet [4]. Die PUVA-Therapie ist insbesondere bei Mycosis fungoides (bei Frühformen auch die NB-UVB) und Sezary-Syndrom eine wichtige Therapieoption und kann den oft ausgeprägten Pruritus signifikant vermindern [23]. Auch bei der Urticaria pigmentosa, der kutanen Variante der Mastozytose, kann die PUVA-Therapie und, falls verfügbar, die UVA-1-Therapie, zu einer Stabilisierung bzw. einer Reduktion der Zahl der Mastzellen in der Haut und wahrscheinlich dadurch auch zu einer Reduktion des Pruritus führen [4].

Chronischer Pruritus ohne primäre Hautveränderungen (Gruppe II der IFSI-Klassifikation)

Chronischer Pruritus kann auch bei sonst unauffälliger Haut auftreten. Vor allem bei generalisierten Formen des chronischen Pruritus finden sich oft internistische Ursachen wie ausgeprägte Niereninsuffizienz (v. a. hämodialysepflichtige Niereninsuffizienz), cholestatische Lebererkrankungen (z. B. die primär biliäre Cholangitis) und hämatologische Erkrankungen (z. B. Polycythaemia vera oder Hodgkin-Lymphome), aber auch Stoffwechselerkrankungen wie Hypound Hyperthyreose, Diabetes mellitus und Eisenmangel oder medikamentöse Ursachen (z.B. Hydroxyethylstärke, Chloroquine; [8]). Die Phototherapie kann in allen diesen Fällen von chronischem Pruritus versucht werden, wenn die Behandlung der Grundkrankheiten zu keiner ausreichenden Reduktion des Pruritus führt oder nicht möglich ist, weil keine spezifische Therapie zur Verfügung steht bzw. die Ursache nicht bekannt ist („chronisch idiopathischer Pruritus").

Wie bereits von Gilchrest et al. [22, 23] beschrieben, hat $\mathrm{BB}($,broadband“)UVB eine signifikante antipruritische Wirkung bei hämodialysepflichtigen Patienten und führt in vielen Fällen zu einer deutlichen Verbesserung der Lebensqualität der Betroffenen. Natürlich können auch nichtdialysepflichtige Patienten mit eingeschränkter Nierenfunktion erfolgreich behandelt werden. Für NB-UVB hat es in der Literatur unterschiedliche Berichte in Bezug auf seine antipruri- tische Wirkung bei niereninsuffizienten Patienten gegeben. Eine Studie konnte keinen signifikanten Effekt von NB-UVB im Vergleich zu UVA finden [24]. Eine andere Studie bei chronisch niereninsuffizienten Patienten mit oder ohne Hämodialyse ergab eine signifikante Reduktion des Pruritus durch NB-UVB im Mittel um mehr als 50\% innerhalb von 8 Wochen. Bei dieser Studie zeigten auch Patienten mit idiopathischem, chronischem Pruritus eine signifikante Reduktion des Pruritus in gleicher Weise durch NB-UVB [25]. Wie schon Gilchrest et al. konnten auch diese Autoren eine Reduktion des Pruritus bei ihren Patienten nach der 2 . Woche bemerken, also etwas verzögert. In einer weiteren Studie wurde die Wirksamkeit von NB-UVB auf chronischen Pruritus bei niereninsuffizienten Patienten belegt [26]. Auch in unserer Erfahrung kann NB-UVB bei niereninsuffizienten Patienten mit und ohne Hämodialyse chronischen Pruritus signifikant reduzieren [27] und evtl. vorhandene sekundäre Kratzeffloreszenzen zur Abheilung bringen (• Abb. 1).

Hepatobiliäre Erkrankungen (mit und ohne Cholestase), wie z. B. die primär biliäre Cholangitis, andere Cholangitiden, Schwangerschaftscholestase und Hepatitis $C$, gehen oft mit chronischem Pruritus einher. Auch nach Beseitigung der Leberfunktionsstörung und Ausschöpfung der leitliniengerechten Therapien wie Cholestyramin, $\mu$-Opiatantagonisten, Rifampicin und Sertralin bleibt oft ein quälender Pruritus zurück. In diesen Fällen sollte zumindest eine UVB-Phototherapie versucht werden [28], deren Wirksamkeit in einzelnen Studien und Fallberichten beschrieben wurde [29]. Insbesondere bei der Schwangerschaftscholestase kann die UVB-Therapie versucht werden, um Pruritus und Hautveränderungen zu bessern oder zu beseitigen, wenn Ursodesoxycholsäure, Antihistaminika und Lokaltherapie nicht ausreichend wirken [30].

Die Phototherapie ist auch in vielen Fällen bei Patienten mit chronischem Pruritus bei hämatologischen Erkrankungen wirksam. Bei Polycythaemia vera kann der quälende, oft ",aquagene“ Pruritus durch Phototherapie mit BBoder NB-UVB deutlich reduziert werden 
oder komplett verschwinden [31]. Auch bei Hodgkin-Lymphom wurde die UVBTherapie erfolgreich zur Linderung des Pruritus eingesetzt [32].

\section{Chronische Prurigo und Patienten mit sekundären Kratzeffloreszenzen}

Wie bereits oben beschrieben, sind der chronische Pruritus und die Hautveränderungen bei chronischer Prurigo [6] eine besondere therapeutische Herausforderung. Eine Abheilung der chronischen Prurigo gelingt üblicherweise nur durch eine lang dauernde weitgehende Unterdrückung des chronischen Pruritus und möglicherweise einer zusätzlichen entzündungshemmenden Therapie. Nachdem es bisher keine zugelassenen wirksamen Medikamente gegen die chronische Prurigo gibt, ist der Einsatz der Phototherapie aus meiner Sicht frühzeitig zu erwägen, wenn lokaltherapeutische antipruritische Maßnahmen keine Besserung bringen.

In einem kürzlich erschienenen Review von Nakamura und Koo [33], in dem die Literatur von 1980 bis 2015 über Photo(chemo)therapien bei „Prurigo nodularis" zusammengefasst wurde, zeigten sich sämtliche Phototherapien (BB-UVB, NB-UVB, orale und BadePUVA, UVA und auch Excimer 308-nmLaser) als wirksam. In vielen Fällen wurde eine deutliche Besserung sowohl des Pruritus als auch der pruriginösen Hautveränderungen beobachtet. Auch die Kombinationsmöglichkeiten mit anderen systemischen (z. B. Thalidomid) und topischen Medikamenten (z. B. Glukokortikosteroidexterna), sowie zweier unterschiedlicher Photo(chemo)therapien (z. B. Ganzkörperphototherapie plus lokale Excimer-Laser-Therapie besonders hartnäckiger Prurigoknoten) wurde hervorgehoben.

Limitationen der Aussagekraft vieler Studien in der Photodermatologie sind aber die oft geringe Patientenzahl und die fehlenden Vergleiche der einzelnen Phototherapiemodalitäten untereinander, sodass nicht immer eine ausreichend gesicherte Empfehlung für die eine oder andere UV-Bestrahlung gegeben werden kann. In der Therapie der chronischen Prurigo (v. a. bei nodulärem $\mathrm{Typ}=$ Prurigo nodularis) haben sich in der Praxis aber die BB-UVB und NB-UVB-Therapien etabliert. Diese Therapien sind in vielen hautärztlichen Ordinationen verfügbar, sie sind sehr gut verträglich, und viele der niedergelassenen Dermatologen sind mit deren Durchführung sehr gut vertraut. In einer Vergleichsstudie konnten wir zeigen, dass bei Patienten mit chronischem Pruritus (meist mit chronischer Prurigo oder sekundären Kratzeffloreszenzen) sowohl die BB-UVB als auch die NBUVB chronischen Pruritus und pruriginöse Hautveränderungen signifikant reduzierten und die NB-UVB-Therapie der BB-UVB-Therapie nicht unterlegen ist [30]. Dies ist insofern von praktischer Bedeutung, da in älteren Hautarztpraxen noch Bestrahlungsgeräte mit BBUVB-Röhren (meist „selektive UV-Phototherapie" [SUP]) stehen, in neueren Hautarztpraxen aber fast ausschließlich NB-UVB-Geräte zu finden sind. Beide Bestrahlungsquellen sind daher unter Berücksichtigung der unterschiedlichen Dosisbereiche für die Behandlung von Patienten mit chronischer Prurigo oder sekundären Kratzeffloreszenzen sehr gut einsetzbar.

\section{》) Die NB-UVB-Therapie ist} der BB-UVB-Therapie nicht unterlegen

Sollte die UVB-Therapie nicht ausreichend wirksam sein, kann die orale PUVA oder Ganzkörper-Bade-PUVA eingesetzt werden. Wenn diese Therapie nicht in der eigenen Praxis durchgeführt wird, können die Patienten an entsprechende Zentren zugewiesen werden.

\section{Einsatz der Phototherapie in den verschiedenen Altersgruppen}

Neben dem breiten Indikationsspektrum der Phototherapie bei Erkrankungen, die mit chronischem Pruritus einhergehen, hat die Phototherapie auch den Vorteil, dass sie bei Patienten nahezu jeden Lebensalters durchgeführt werden kann. Bei atopischer Dermatitis ist der Einsatz bereits ab dem Kindesalter möglich und sehr gut wirksam [34, 35]. Ein absolutes Mindestalter der Kinder für die Phototherapie gibt es nicht, und sofern die Kinder den erforderlichen Maßnahmen Folge leisten können (ruhiges Stehen in der UV-Kabine, Tragen der Schutzbrillen etc.), ist eine Phototherapie bei Kindern möglich. Die Indikation für eine Phototherapie im Kindesalter ist allerdings streng $\mathrm{zu}$ stellen (z. B. unzureichendes Ansprechen auf eine bereits optimierte Lokaltherapie). Die UV-Dosis sollte bei Kindern möglichst gering gehalten (UV-Erytheme müssen vermieden werden!) und die Therapiedauer so kurz wie möglich gewählt werden (keine Langzeit- oder Erhaltungstherapien). Bei richtiger Anwendung kann die Phototherapie im Kindesalter über Phasen einer schubhaften Verschlechterung einer atopischen Dermatitis hinweghelfen, ohne systemische Medikamente wie Cyclosporin einsetzen zu müssen. Auch bei der Psoriasis im Kindesalter (v.a. vom Guttata-Typ), die nach Infekten wie Angina tonsillaris auftreten kann, ist die Phototherapie sehr gut wirksam.

\section{》) Die Indikation für eine Phototherapie im Kindesalter ist streng zu stellen}

In der Schwangerschaft und Stillperiode, in denen systemische Medikamente meist kontraindiziert sind, ist die Phototherapie eine wertvolle Behandlungsmöglichkeit bei chronischem Pruritus. Vor allem bei einem Aufflammen einer atopischen Dermatitis oder Psoriasis in der Schwangerschaft oder der Schwangerschaftscholestase sollte die Phototherapie versucht werden, wenn spezifische oder lokale Therapien dieser Dermatosen nicht ausreichen [31]. Bei der Phototherapie in der Schwangerschaft (v.a. Frühschwangerschaft) sollten die Frauen die üblicherweise in der Schwangerschaft empfohlenen Folsäurepräparate einnehmen [36].

Besonders im höheren Lebensalter, in dem auch mit einer erhöhten Inzidenz von chronischem Pruritus bei Haut- und systemischen Erkrankungen zu rechnen ist (s. auch Beitrag von 
Weishaar und Mettang in diesem Heft), ist der Einsatz der Phototherapie als antipruritische Therapie sehr gut geeignet. Eine ausreichende Mobilität des Patienten vorausgesetzt, ist die Phototherapie eine sehr wirkungsvolle und nebenwirkungsarme Therapiemodalität, die meist zu einer deutlichen Reduktion oder zum Verschwinden des Pruritus führt. Eine sorgfältige Aufklärung, Hautuntersuchung auf aktinische Schäden oder Hauttumoren und die genaue Anamnese der eingenommenen $\mathrm{Me}$ dikamente, Nahrungsergänzungsmittel und ggf. Heilkräuter sind gerade in dieser Altersgruppe von großer Bedeutung. Insbesondere ältere Patienten nehmen oft eine größere Anzahl dieser Mittel ein. Für die Phototherapie sind bei der Anamnese v. a. potenziell phototoxische Medikamente und immunsuppressive Medikamente von besonderer Bedeutung, da sie möglicherweise (relative) Kontraindikationen für die Phototherapie darstellen. Ein Beispiel ist das Johanniskraut (Hypericum perforatum, engl. „St. John's wort“), das oft bei milden depressiven Zuständen eingesetzt wird, von den Patienten aber nicht als Medikament wahrgenommen und daher bei der Anamnese oft „verschwiegen“ wird. Das enthaltende Hypericin senkt die Erythemschwelle im gesamten UVBereich und wird auch durch sichtbares Licht aktiviert. Ausgeprägte phototoxische Reaktionen bei jeder UV-Therapie können die Folge sein [37]. Daneben sind aber noch andere potenziell phototoxische Medikamente aus den Gruppen der Antibiotika, nichtsteroidale Antirheumatika (NSAID), Antihypertensiva, Diuretika und Chemotherapeutika zu berücksichtigen.

Wichtig ist in dieser Altersgruppe auch, die UV-Dosis am Anfang und bei den Steigerungen niedriger zu wählen, da durch die geringere Pigmentierungskapazität eine erhöhte Lichtempfindlichkeit resultieren kann. Ältere Patienten müssen während der Phototherapie engmaschiger kontrolliert werden. Außerdem brauchen sie mehr Zeit für die Therapie, da das Ausziehen und Ankleiden länger dauert. Dennoch ist die Phototherapie bei älteren Patienten als sehr gut wirksam und nebenwirkungsarm einzustufen und eine wichtige Säule in der Behandlung des chronischen Pruritus bei Älteren [38].

\section{Nebenwirkungen durch die Phototherapie}

Bei richtiger und sorgfältiger Durchführung sind die Nebenwirkungen einer Phototherapie [4] gering, wenn man von Übelkeit und Schwindel durch die Einnahme von Oxsoralen ${ }^{\circledR}$ im Rahmen einer PUVA-Therapie absieht. UV-Erytheme, die bei stärkerer Ausprägung auch zur Verstärkung des Pruritus führen können, sind die häufigsten akuten Nebenwirkungen. Selten kann es zur Auslösung einer polymorphen Lichtdermatose („Sonnenallergie“) oder zu einer Aggravierung einer bestehenden atopischen Dermatitis oder Psoriasis kommen. Bei sorgfältiger Anamnese zu ähnlichen Ereignissen in der Vergangenheit sind diese meist zu vermeiden. Mögliche phototoxische Reaktionen durch Einnahme von photosensibilisierenden Substanzen (neu verschriebene Medikamente oder nichtwissentlicher Konsum bestimmter Nahrungsmittel oder Pflanzenextrakte) sind selten, können aber vorkommen.

\section{) UV-Erytheme sind die häufigsten akuten Nebenwirkungen}

Langzeitnebenwirkungen der Phototherapien wie beschleunigte Hautalterung und gesteigertes Hauttumorrisikos, die v. a. bei nahezu jährlichen, wiederholten Behandlungen von Psoriasispatienten auftreten können, sind bei der Behandlung von chronischem Pruritus unterschiedlicher Genese wenig zu fürchten, da die Anzahl der Bestrahlungen und die kumulativen UV-Dosen in der Regel deutlich geringer sind.

\section{Fazit für die Praxis}

- Die Phototherapie ist eine wirksame Behandlung gegen chronischen Pruritus bei unterschiedlichen entzündlichen Hautkrankheiten und systemischen Erkrankungen.
- Es können Erwachsene aller Altersstufen, Schwangere und stillende Mütter, Jugendliche und unter strengerer Indikationsstellung auch Kinder mit UV-Licht behandelt werden.

- Wenn photosensibilisierende Substanzen gemieden werden, sind bei der Phototherapie keine akuten negativen Interaktionen mit lokalen oder systemischen Therapien zu befürchten.

- Bei richtiger Anwendung sind die Nebenwirkungen durch eine Phototherapie bei chronischem Pruritus sehr selten und gering ausgeprägt.

- Die Phototherapie sollte bei chronischem Pruritus früh eingesetzt werden, wenn lokaltherapeutische Maßnahmen versagen.

- Die phototherapeutische Aus- und Weiterbildung der Dermatologinnen und Dermatologen sollte aufgrund der vielfältigen Einsatzmöglichkeiten der Phototherapie in der Dermatologie ein fixer Bestandteil der Facharztausbildung sein.

\section{Korrespondenzadresse}

ao. Univ. Prof. Dr. F. J. Legat

Universitätsklinik für Dermatologie und Venerologie, Medizinische Universität Graz Auenbruggerpl. 8, 8036 Graz, Österreich franz.legat@medunigraz.at

Funding. Open access funding provided by Medical University of Graz.

\section{Einhaltung ethischer Richtlinien}

Interessenkonflikt. F.J. Legat gibt an, dass kein Interessenkonflikt besteht.

Dieser Beitrag beinhaltet keine vom Autor durchgeführten Studien an Menschen oder Tieren. Alle Patienten, die über Bildmaterial oder anderweitige Angaben innerhalb des Manuskripts zu identifizieren sind, haben hierzu ihre schriftliche Einwilligung gegeben. Im Falle von nicht mündigen Patienten liegt die Einwilligung eines Erziehungsberechtigten oder des gesetzlich bestellten Betreuers vor.

Open Access. Dieser Artikel wird unter der Creative Commons Namensnennung 4.0 International Lizenz (http://creativecommons.org/licenses/by/4.0/deed. de) veröffentlicht, welche die Nutzung, Vervielfältigung, Bearbeitung, Verbreitung und Wiedergabe in jeglichem Medium und Format erlaubt, sofern Sie den/die ursprünglichen Autor(en) und die Quelle ordnungsgemäß nennen, einen Link zur Creative Com- 
mons Lizenz beifügen und angeben, ob Änderungen vorgenommen wurden.

\section{Literatur}

1. Møller KI, Kongshoj B, Philipsen PA et al (2005) How Finsen's light cured lupus vulgaris. Photodermatol Photoimmunol Photomed 21:118-124

2. Pathak MA, Fitzpatrick TB (1992) The evolution of photochemotherapy with psoralens and UVA (PUVA): 2000 BC to 1992 AD. J Photochem Photobiol B, Biol 14:3-22

3. Lim HW, Silpa-archa N, Amadi U et al (2015) Phototherapy in dermatology: a call for action. J Am Acad Dermatol 72:1078-1080

4. Herzinger $T$, Berneburg $M$, Ghoreschi $K$ et al (2016) S1-Leitlinie zur UV-Phototherapie und Photochemotherapie 14, S853-876

5. Ständer S, Weisshaar E, Mettang T et al (2007) Clinical classification of itch: a position paper of the international forum for the study of itch. Acta Derm Venereol 87:291-294

6. Pereira MP,SteinkeS,ZeidlerCetal(2017) European academy of dermatology and venereology European prurigo project: expert consensus on the definition, classification and terminology of chronic prurigo. J Eur Acad Dermatol Venereol. https://doi.org/10.1111/jdv.14570

7. Matterne $U$, Apfelbacher $\mathrm{CJ}$, Loerbroks $\mathrm{A}$ et al (2011) Prevalence, correlates and characteristics of chronic pruritus: a population-based crosssectional study. Acta Derm Venereol 91:674-679

8. Ständer S, Zeidler C, Augustin M et al (2017) S2k guidelines for the diagnosis and treatment of chronic pruritus - update-short version. J Dtsch Dermatol Ges 15:860-872. https://doi.org/10. $1111 /$ ddg. 13304

9. Meng J, SteinhoffM (2016) Molecular mechanisms of pruritus. Curr Res Transl Med 64:203-206

10. Théréné $C$, Brenaut $E$, Barnetche T et al (2018) Efficacy of systemic treatments of psoriasis on pruritus: a systemic literature review and metaanalysis. J Invest Dermatol 138:38-45. https://doi. org/10.1016/j.jid.2017.05.039

11. Garritsen FM, Brouwer MWD, Limpens J et al (2014) Photo(chemo)therapy in the management of atopic dermatitis: an updated systematic review with implications for practice and research. $\mathrm{Br} J$ Dermatol 170:501-513

12. Rivard J, Lim HW (2005) Ultraviolet phototherapy for pruritus. Dermatol Ther 18:344-354

13. Mohammad T, Morrison H, Hoengesch H (1999) Urocanic acid photochemistry and photobiology. Photochem Photobiol 69:115-135

14. Schmelz M (2015) Neurophysiology and itch pathways. Handb Exp Pharmacol 226:39-55. https://doi.org/10.1007/978-3-662-44605-8_3

15. Schwarz T, Beissert S (2013) Milestones in Photoimmunology. J Invest Dermatol 133:E7-E10

16. Legat FJ, Wolf $P$ (2006) Photodamage to the cutaneous sensory nerves: role in photoaging and carcinogenesis of the skin? Photochem Photobiol Sci 5:170-176

17. Legat FJ, Wolf P (2009) Cutaneous sensory nerves: mediators of phototherapeutic effects? Front Biosci (LandmarkEd) 14:4921-4931

18. Stull C, Lavery MJ, Yosipovitch G (2016) "Immunosuppressants". In: Misery L, Ständer S (Hrsg) Pruritus. Springer, London, S 379-406 https://doi. org/10.1007/978-3-319-33142-3_52

19. Gilchrest BA, Rowe JW, Brown RS et al (1977) Relief of uremic pruritus with ultraviolet phototherapy. NEngl J Med 297:136-138
20. Gilchrest BA, Rowe JW, Brown RS et al (1979) Ultraviolet phototherapy of uremic pruritus. Longterm results and possible mechanism of action. Ann Intern Med 91:17-21

21. Legat FJ, Hofer A, Brabek Eet al (2003) Narrowband UV-B vs medium-dose UV-A1 phototherapy in chronic atopic dermatitis. Arch Dermatol 139:223-224

22. Evers AWM, Kleinpenning MM, Smits $T$ et al (2009) Itch and scratching as predictors of time to clearance of psoriasis with narrow-band ultraviolet B therapy. Br JDermatol 161:542-546

23. Olsen EA, Hodak E, Anderson T et al (2016) Guidelines for phototherapy of mycosis fungoides and Sézary syndrome: A consensus statement of the United States Cutaneous Lymphoma Consortium. J Am Acad Dermatol 74:27-58. https://doi.org/10.1016/j.jaad.2015.09.033

24. Ko MJ, Yang JY, Wu HY (2011) Narrowband ultraviolet $\mathrm{B}$ phototherapy for patients with refractory uraemic pruritus: a randomized controlled trial. $\mathrm{Br}$ JDermatol 165:633-639

25. Seckin D, Demircay Z, Akin O (2007) Generalized pruritus treated with narrowband UVB. Int $J$ Dermatol 46:367-370

26. Wang TJ, Lan LC, Lu CS et al (2014) Efficacy of narrowband ultraviolet phototherapy on renal pruritus.J Clin Nurs 23:1593-1602. https://doi.org/ 10.1111/jocn.12252

27. Legat FJ, Hofer $A$, Gruber-Wackernagel $A$ et al (2017) Both narrowband-UVB and broadbandUVB are equally effective in reducing itch in chronic pruritus patients. Abstract, Poster N74, 9th World congress on itch (WCI), Wraclaw, 15.-17.10.2017, Poland.https://medicaljournals.se/wci/

28. Kremer AE, Bolier R, van DijkRetal (2014) Advances in pathogenesis and management of pruritus in cholestasis. Dig Dis 32:637-645. https://doi.org/ 10.1159/000360518

29. Decock S, Roelandts R, Van Steenbergen W et al (2012) Cholestasis-induced pruritus treated with ultraviolet B phototherapy: an observational case series study. J Hepatol 57:637-641

30. Lambert J (2016) Itch in pregnancy management Curr Probl Dermatol 50:164-172. https://doi.org/ 10.1159/000446087

31. Baldo A, Sammarco E, Plaitano R et al (2002) Narrowband (TL01) ultraviolet B phototherapy for pruritus in polycythemia vera. Br J Dermatol 147:979-981

32. Kaptanoglu AF, Oskay T (2003) Ultraviolet B treatment for pruritus in Hodgkin's lymphoma. J Eur Acad Dermatol Venereol 17:489-490

33. Nakamura M, Koo JYM (2016) Phototherapy for the treatment of prurigo nodularis: a review. Dermatol Online J22(4):pii: 13030/qt4b07778z

34. Dayal S, Pathak K, Sahu P, Jain VK (2016) Narrowband UV-B phototherapy in childhood atopic dermatitis: efficacy and safety. An Bras Dermatol 92:801-806. https://doi.org/10.1590/ abd1806-4841.20175958

35. Wollenberg A, Barbarot S, Bieber T et al (2018) Consensus-based European guidelines for treatment of atopic eczema (atopic dermatitis) in adults and children: part I. J Eur Acad Dermatol Venereol 32:657-682

36. Zhang M, Goyert G, Lim HW (2017) Folate and phototherapy: what should we inform our patients? J Am Acad Dermatol 77:958-964. https:// doi.org/10.1016/j.jaad.2016.10.016

37. Beattie PE, Dawe RS, Traynor NJ et al (2005) Can St John's wort (hypericin) ingestion enhance the erythemal response during high-dose ultraviolet A1 therapy? Br JDermatol 153:1187-1191
38. Powell JB, Gach JE (2015) Phototherapy in the elderly. Clin Exp Dermatol 40:605-610 